\title{
FAMILIES OF FRACTIONAL FANTAPPIÈ TRANSFORMS EVGUENI DOUBTSOV
}

\author{
(Received 23 September 2009)
}

\begin{abstract}
Let $B_{n}$ denote the unit ball in $\mathbb{C}^{n}, n \geq 1$. Given an $\alpha>0$, let $\mathcal{F}_{\alpha}(n)$ denote the class of functions defined for $z \in B_{n}$ by integrating the kernel $(1-\langle z, w\rangle)^{-\alpha}$ against a complex Borel measure $d \mu(w), w \in B_{n}$. The family $\mathcal{F}_{0}(n)$ corresponds to the logarithmic kernel $\log (1 /(1-\langle z, w\rangle))$. Various properties of the spaces $\mathcal{F}_{\alpha}(n), \alpha \geq 0$, are obtained. In particular, pointwise multiplies for $\mathcal{F}_{\alpha}(n)$ are investigated.
\end{abstract}

2000 Mathematics subject classification: primary 32A26; secondary 32A37, 46E15, 46J15.

Keywords and phrases: fractional Fantappiè transform, pointwise multiplier.

\section{Introduction}

Let $B_{n}=\left\{z \in \mathbb{C}^{n}:|z|<1\right\}, n \geq 1$. For $\lambda \in B_{1}$, put

$$
\begin{aligned}
& k_{0}(\lambda)=\log \frac{1}{1-\lambda} ; \\
& k_{\alpha}(\lambda)=\frac{1}{(1-\lambda)^{\alpha}}, \quad \alpha>0 .
\end{aligned}
$$

Here and in what follows we use the principal branch of the logarithm.

1.1. Fractional Fantappiè transforms. Let $\mathcal{M}\left(B_{n}\right)$ denote the space of complexvalued Borel measures defined on the ball $B_{n}$. Let $\alpha \geq 0$. Given a measure $\mu \in$ $\mathcal{M}\left(B_{n}\right)$, its fractional Fantappiè transform of order $\alpha$ is defined by the identity

$$
F_{\alpha}[\mu](z)=\int_{B_{n}} k_{\alpha}(\langle z, w\rangle) d \mu(w), \quad z \in B_{n} .
$$

The classical Fantappiè transform in the ball is $F_{1}[\mu]$. See, for example, [1] for a detailed treatment of the Fantappiè transform.

Note that

$$
\frac{1}{(1-\langle z, w\rangle)^{n+1}}, \quad z, w \in B_{n},
$$

This research was supported by RFBR (grant no. 08-01-00358-a).

(C) 2010 Australian Mathematical Publishing Association Inc. 0004-9727/2010 \$16.00 
is the Bergman kernel in the ball. So, one may consider $F_{\alpha}[\mu]$ as fractional Bergman transforms of $\mu \in \mathcal{M}\left(B_{n}\right)$.

Let $v_{n}$ denote the normalized Lebesgue measure on the ball $B_{n}$. In numerous applications one usually considers $F_{\alpha}[\mu]$, where $\mu$ is absolutely continuous with respect to $v_{n}$ (see, for example, [12]).

Let $\mathcal{H o l}\left(B_{n}\right)$ denote the space of functions holomorphic on the ball $B_{n}$. Consider the families

$$
\begin{aligned}
& \mathcal{F}_{0}(n)=\left\{f \in \mathcal{H} \operatorname{ol}\left(B_{n}\right): f-f(0)=F_{0}[\mu] \text { for some } \mu \in \mathcal{M}\left(B_{n}\right)\right\} \\
& \mathcal{F}_{\alpha}(n)=\left\{F_{\alpha}[\mu]: \mu \in \mathcal{M}\left(B_{n}\right)\right\}, \quad \alpha>0 .
\end{aligned}
$$

Note that

$$
1=\int_{B_{n}} \frac{d v_{n}(w)}{(1-\langle z, w\rangle)^{\alpha}} \quad \forall z \in B_{n}, \alpha>0 .
$$

Therefore, $1 \in \mathcal{F}_{\alpha}(n)$ for all $\alpha \geq 0$. Standard arguments show that $\mathcal{F}_{\alpha}(n), \alpha \geq 0$, are Banach spaces with respect to the following norms:

$$
\begin{aligned}
& \|f\|_{\mathcal{F}_{0}(n)}=|f(0)|+\inf \left\{\|\mu\|_{\mathcal{M}\left(B_{n}\right)}: f-f(0)=F_{0}[\mu]\right\}, \quad f \in \mathcal{F}_{0}(n) ; \\
& \|f\|_{\mathcal{F}_{\alpha}(n)}=\inf \left\{\|\mu\|_{\mathcal{M}\left(B_{n}\right)}: f=F_{\alpha}[\mu]\right\}, \quad f \in \mathcal{F}_{\alpha}(n), \alpha>0 .
\end{aligned}
$$

Assume that $f=F_{\alpha}[\mu], \alpha>0$, for some positive measure $\mu \in \mathcal{M}\left(B_{n}\right)$. Let $f=$

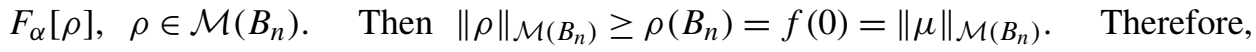
$\|f\|_{\mathcal{F}_{\alpha}(n)}=\|\mu\|_{\mathcal{M}\left(B_{n}\right)}$.

1.2. Multipliers. Assume that $X, Y \subset \mathcal{H o l}\left(B_{n}\right)$ are Banach spaces. A function $g \in \mathcal{H} \operatorname{col}\left(B_{n}\right)$ is called a (pointwise) multiplier from $X$ to $Y$ provided that $f g \in Y$ for all $f \in X$. Let $\mathfrak{M}(X, Y)$ denote the set of all multipliers from $X$ to $Y$. Put $\mathfrak{M}(X)=\mathfrak{M}(X, X)$ and $\mathfrak{M}_{\alpha}(n)=\mathfrak{M}\left(\mathcal{F}_{\alpha}(n)\right)$. $\mathfrak{M}_{\alpha}(n), \alpha \geq 0$, is a Banach space with respect to the natural operator norm defined by the identity

$$
\|g\|_{\mathfrak{M}_{\alpha}(n)}=\sup \left\{\|f g\|_{\mathcal{F}_{\alpha}(n)}:\|f\|_{\mathcal{F}_{\alpha}(n)} \leq 1\right\}, \quad g \in \mathfrak{M}_{\alpha}(n) .
$$

1.3. Families $\mathcal{F}_{\boldsymbol{\alpha}}(\boldsymbol{n})$ and fractional Cauchy transforms. Let $\mathcal{M}\left(\partial B_{n}\right)$ denote the space of complex-valued Borel measures defined on the sphere $\partial B_{n}$. Let $\alpha \geq 0$. Given a measure $\rho \in \mathcal{M}\left(\partial B_{n}\right)$, its fractional Cauchy transform of order $\alpha$ is defined by the identity

$$
K_{\alpha}[\rho](z)=\int_{\partial B_{n}} k_{\alpha}(\langle z, \zeta\rangle) d \rho(\zeta), \quad z \in B_{n} .
$$

Put

$$
\begin{aligned}
& \mathcal{K}_{0}(n)=\left\{f \in \mathcal{H} \operatorname{ol}\left(B_{n}\right): f-f(0)=K_{0}[\rho] \text { for some } \rho \in \mathcal{M}\left(\partial B_{n}\right)\right\} \\
& \mathcal{K}_{\alpha}(n)=\left\{K_{\alpha}[\rho]: \rho \in \mathcal{M}\left(\partial B_{n}\right)\right\}, \quad \alpha>0 .
\end{aligned}
$$

$\mathcal{F}_{\alpha}(n), \alpha \geq 0$, are Banach spaces with respect to the natural norms

$$
\begin{aligned}
& \|f\|_{\mathcal{K}_{0}(n)}=|f(0)|+\inf \left\{\|\rho\|_{\mathcal{M}\left(\partial B_{n}\right)}: f-f(0)=K_{0}[\rho]\right\}, \quad f \in \mathcal{K}_{0}(n) ; \\
& \|f\|_{\mathcal{K}_{\alpha}(n)}=\inf \left\{\|\rho\|_{\mathcal{M}\left(\partial B_{n}\right)}: f=K_{\alpha}[\rho]\right\}, \quad f \in \mathcal{K}_{\alpha}(n), \alpha>0 .
\end{aligned}
$$


The families $\mathcal{K}_{\alpha}(n)$ and $\mathfrak{M}\left(\mathcal{K}_{\alpha}(n)\right)$ are rather close to $\mathcal{F}_{\alpha}(n)$ and $\mathfrak{M}_{\alpha}(n)$, respectively. In particular, $\mathcal{F}_{\alpha}(n) \subset \mathcal{K}_{\alpha}(n), \mathcal{F}_{\alpha}(n) \neq \mathcal{K}_{\alpha}(n)$ and $\mathfrak{M}_{\alpha}(n) \subset \mathfrak{M}\left(\mathcal{K}_{\alpha}(n)\right)$. The author does not know whether $\mathfrak{M}_{\alpha}(n)$ coincides with $\mathfrak{M}\left(\mathcal{K}_{\alpha}(n)\right)$.

The classical spaces $\mathcal{K}_{1}(1)$ and $\mathfrak{M}\left(\mathcal{K}_{1}(1)\right)$ are investigated in [3]. Various properties of the families $\mathcal{K}_{\alpha}(1)$ and $\mathfrak{M}\left(\mathcal{K}_{\alpha}(1)\right)$ are collected in [8]. Certain results about $\mathcal{K}_{\alpha}(n)$ and $\mathfrak{M}\left(\mathcal{K}_{\alpha}(n)\right)$ are obtained in [4-6] for arbitrary $n \in \mathbb{N}$. To the best of the author's knowledge, the spaces $\mathcal{F}_{\alpha}(n)$ and $\mathfrak{M}_{\alpha}(n)$ have not been investigated systematically.

1.4. Organization of the paper. Definitions and auxiliary results are collected in Section 2. In Section 3 embedding properties for the families $\mathcal{F}_{\alpha}(n)$ and BergmanSobolev spaces are investigated. Radial derivatives of functions from $\mathcal{F}_{\alpha}(n)$ are studied in Section 4. It is shown in Section 5 that $\mathcal{F}_{\alpha}(n)$ is a proper subset of $\mathcal{K}_{\alpha}(n)$. The multiplier spaces $\mathfrak{M}_{\alpha}(n)$ are investigated in the final Sections 6 and 7. The main results are Proposition 7.1 and Theorem 7.7.

\section{Preliminaries}

2.1. Radial derivatives. Given $f \in \mathcal{H}$ ol $\left(B_{n}\right)$, the radial derivative $\mathcal{R} f$ is defined by the identity

$$
\mathcal{R} f(z)=\sum_{j=1}^{n} z_{j} \frac{\partial f}{\partial z_{j}}(z), \quad z \in B_{n}
$$

It is well known that

$$
f(z)-f(0)=\int_{0}^{1} \frac{\mathcal{R} f(t z)}{t} d t, \quad z \in B_{n} .
$$

Direct calculations show that

$$
\mathcal{R}(f g)=f \cdot \mathcal{R} g+\mathcal{R} f \cdot g \quad \forall f, g \in \mathcal{H} \text { ol }\left(B_{n}\right) .
$$

Further, put

$$
R=\mathcal{R}+I: \mathcal{H o l}\left(B_{n}\right) \rightarrow \mathcal{H o l}\left(B_{n}\right)
$$

Note that

$$
R(f g)=f \cdot R g+\mathcal{R} f \cdot g \quad \forall f, g \in \mathcal{H} o l\left(B_{n}\right) .
$$

2.2. Operators of radial differentiation. Fractional analogs of the operator $R$ are defined in terms of the homogeneous expansions of holomorphic functions. Namely, assume that $s, t \in \mathbb{R}, s>-n-1$ and $s+t>-n-1$. An invertible operator

$$
D_{s}^{t}: \mathcal{H o l}\left(B_{n}\right) \rightarrow \mathcal{H o l}\left(B_{n}\right)
$$

is defined as follows. If $f=\sum_{k=0}^{\infty} f_{k}$ is the homogeneous expansion of $f \in \mathcal{H} \operatorname{Hol}\left(B_{n}\right)$, then

$$
D_{s}^{t} f(z)=\sum_{k=0}^{\infty} \frac{\Gamma(n+1+s) \Gamma(n+1+k+s+t)}{\Gamma(n+1+s+t) \Gamma(n+1+k+s)} f_{k}(z), \quad z \in B_{n} .
$$

Note that, under the above restrictions on $s$ and $t$, definition (2.3) coincides with those in [12, Section 1.4] and [9]. 
We have

$$
\left(D_{s}^{t}\right)^{-1}=D_{s+t}^{-t}: \mathcal{H} \operatorname{ol}\left(B_{n}\right) \rightarrow \mathcal{H o l}\left(B_{n}\right),
$$

where $D_{s+t}^{-t}$ is defined by formula (2.3). Finally, observe that $R=\mathcal{R}+I=D_{-n}^{1}$.

\section{Embedding properties}

3.1. Weighted Bergman spaces. Recall that $v_{n}$ denotes the normalized Lebesgue measure on the ball $B_{n}$. For $p, q>0$, the weighted Bergman space $A_{q}^{p}\left(B_{n}\right)$ consists of those $f \in \mathcal{H} \operatorname{lol}\left(B_{n}\right)$ for which

$$
\|f\|_{A_{q}^{p}\left(B_{n}\right)}^{p}=\int_{B_{n}}|f(z)|(1-|z|)^{q-1} d v_{n}(z)<\infty .
$$

Proposition 3.1. Suppose that $n \in \mathbb{N}$.

(i) If $\alpha>n$, then $A_{\alpha-n}^{1}\left(B_{n}\right) \subset \mathcal{F}_{\alpha}(n)$.

(ii) If $\beta>\alpha \geq 0$ and $\beta>n$, then $\mathcal{F}_{\alpha}(n) \subset A_{\beta-n}^{1}\left(B_{n}\right)$.

PROOF. (i) If $f \in A_{\alpha-n}^{1}\left(B_{n}\right), \alpha>n$, then [12, Theorem 2.2] guarantees that

$$
f(z)=c_{\alpha} \int_{B_{n}} \frac{f(w)\left(1-|w|^{2}\right)^{\alpha-n-1} d v_{n}(w)}{(1-\langle z, w\rangle)^{\alpha}}
$$

for all $z \in B_{n}$. By the assumption,

$$
f(w)\left(1-|w|^{2}\right)^{\alpha-n-1} d v_{n}(w) \in \mathcal{M}\left(B_{n}\right) .
$$

Therefore, $A_{\alpha-n}^{1}\left(B_{n}\right) \subset \mathcal{F}_{\alpha}(n)$ for $\alpha>n$.

(ii) Assume that $\alpha>0, \mu \in \mathcal{M}\left(B_{n}\right)$ and

$$
f(z)=F_{\alpha}[\mu](z)=\int_{B_{n}} \frac{d \mu(w)}{(1-\langle z, w\rangle)^{\alpha}}, \quad z \in B_{n} .
$$

If $\beta>n$ and $\beta>\alpha$, then, changing the order of integration, we obtain

$$
\begin{aligned}
\int_{B_{n}}|f(z)|(1-|z|)^{\beta-n-1} d v_{n}(z) & \leq \int_{B_{n}} \int_{B_{n}} \frac{(1-|z|)^{\beta-n-1} d v_{n}(z)}{|1-\langle z, w\rangle|^{\alpha}} d|\mu|(w) \\
& \leq C \int_{B_{n}} d|\mu|(w)<\infty
\end{aligned}
$$

by [10, Proposition 1.4.10]. So $\mathcal{F}_{\alpha}(n) \subset A_{\beta-n}^{1}\left(B_{n}\right)$. The proof of the embedding $\mathcal{F}_{0}(n) \subset A_{\beta-n}^{1}\left(B_{n}\right), \beta>n$, is analogous.

3.2. Bergman-Sobolev spaces. Suppose that $p, q>0$ and $j \in \mathbb{Z}_{+}$. By definition, the Bergman-Sobolev space $A_{q, j}^{p}\left(B_{n}\right)$ consists of those $f \in \mathcal{H}$ ol $\left(B_{n}\right)$ for which

$$
\|f\|_{A_{q, j}^{p}\left(B_{n}\right)}=\int_{B_{n}}\left|R^{j} f(z)\right|^{p}(1-|z|)^{q-1} d v_{n}(z)<\infty .
$$


For $p \geq 1, A_{q, j}^{p}\left(B_{n}\right)$ is a Banach space. Note that $A_{q, 0}^{p}\left(B_{n}\right)$ coincides with the weighted Bergman space $A_{q}^{p}\left(B_{n}\right)$. Basic properties of the general spaces $A_{q, s}^{p}\left(B_{n}\right)$, $s \in \mathbb{R}$, are gathered together in [2].

Proposition 3.2. Assume that $n \in \mathbb{N}, j \in\{0,1, \ldots, n\}$ and $\alpha>n-j$.

(i) The embedding $A_{\alpha-n+j, j}^{1}\left(B_{n}\right) \subset \mathcal{F}_{\alpha}(n)$ holds.

(ii) If $\beta>\alpha$, then $\mathcal{F}_{\alpha}(n) \subset A_{\beta-n+j, j}^{1}\left(B_{n}\right)$.

ProOf. If $j=0$, then Proposition 3.1 applies. We assume that $j \in\{1, \ldots, n\}$.

(i) Let $f \in A_{\alpha-n+j, j}^{1}\left(B_{n}\right)$. We have

$$
D_{\alpha-n-1}^{j}\left(A_{\alpha-n+j, j}^{1}\left(B_{n}\right)\right)=A_{\alpha-n+j}^{1}\left(B_{n}\right)
$$

by [9, Proposition 3.2]. (Note that in [9], Bergman-Sobolev spaces are called Besov spaces.) Therefore, $D_{\alpha-n-1}^{j} f \in A_{\alpha-n+j}^{1}\left(B_{n}\right) \subset \mathcal{F}_{\alpha+j}(n)$ by Proposition 3.1(i). So,

$$
D_{\alpha-n-1}^{j} f(z)=\int_{B_{n}} \frac{d \mu(w)}{(1-\langle z, w\rangle)^{\alpha+j}}, \quad z \in B_{n},
$$

for some $\mu \in \mathcal{M}\left(B_{n}\right)$. We have $\alpha>0$, hence, the inverse operator $\left(D_{\alpha-n-1}^{j}\right)^{-1}=$ $D_{\alpha+j-n-1}^{-j}$ is correctly defined by (2.3). Next, [12, Proposition 1.14] guarantees that

$$
D_{\alpha+j-n-1}^{-j} \frac{1}{(1-\langle z, w\rangle)^{\alpha+j}}=\frac{1}{(1-\langle z, w\rangle)^{\alpha}}, \quad z, w \in B_{n} .
$$

Therefore,

$$
f(z)=D_{\alpha+j-n-1}^{-j} D_{\alpha-n-1}^{j} f(z)=\int_{B_{n}} \frac{d \mu(w)}{(1-\langle z, w\rangle)^{\alpha}}, \quad z \in B_{n} .
$$

In other words, $f \in \mathcal{F}_{\alpha}(n)$.

(ii) Assume that $j \in\{1, \ldots, n\}, \alpha>n-j$ and

$$
f(z)=\int_{B_{n}} \frac{d \mu(w)}{(1-\langle z, w\rangle)^{\alpha}}, \quad z \in B_{n},
$$

for some $\mu \in \mathcal{M}\left(B_{n}\right)$. For $z \in B_{n}$, straightforward calculations show that

$$
R f(z)=\mathcal{R} f(z)+f(z)=\int_{B_{n}} \frac{\alpha d \mu(w)}{(1-\langle z, w\rangle)^{\alpha+1}}+(1-\alpha) f(z) \in \mathcal{F}_{\alpha+1}(n)+\mathcal{F}_{\alpha}(n) .
$$

By induction,

$$
R^{j} f \in \mathcal{F}_{\alpha+j}(n)+\cdots+\mathcal{F}_{\alpha}(n) .
$$

Now, suppose that $\beta>\alpha$. Note that $\beta+j>\alpha+j$ and $\beta+j>n$, hence,

$$
\mathcal{F}_{\alpha+j}(n)+\cdots+\mathcal{F}_{\alpha}(n) \subset A_{\beta+j-n}^{1}\left(B_{n}\right)
$$

by Proposition 3.1(ii). So (3.1) guarantees that $f \in A_{\beta+j-n, j}^{1}\left(B_{n}\right)$.

For $\alpha=0$, we have the following analog of Proposition 3.2(ii). 
Proposition 3.3. Let $n \in \mathbb{N}$. Then $\mathcal{F}_{0}(n) \subset A_{\varepsilon, n}^{1}\left(B_{n}\right)$ for any $\varepsilon>0$.

PROOF. Let $\mu \in \mathcal{M}\left(B_{n}\right)$ and let

$$
f(z)=f(0)+\int_{B_{n}} \log \frac{1}{1-\langle z, w\rangle} d \mu(w), \quad z \in B_{n} .
$$

For $z \in B_{n}$, direct calculations show that

$$
\begin{aligned}
R f(z)=\mathcal{R} f(z)+f(z) & =\int_{B_{n}} \frac{1}{1-\langle z, w\rangle} d \mu(w)+C+\log \frac{1}{1-\langle z, w\rangle} d \mu(w) \\
& \in \mathcal{F}_{1}(n)+\mathcal{F}_{0}(n) .
\end{aligned}
$$

Therefore,

$$
R^{n} f \in \mathcal{F}_{n}(n)+\cdots+\mathcal{F}_{0}(n) \subset A_{\varepsilon}^{1}\left(B_{n}\right)
$$

by Proposition 3.1(ii) with $\beta=\varepsilon+n>n$. So $f \in A_{\varepsilon, n}^{1}\left(B_{n}\right)$.

Corollary 3.4. Let $n \in \mathbb{N}$ and let $0 \leq \alpha<\beta$. Then $\mathcal{F}_{\alpha}(n) \subset \mathcal{F}_{\beta}(n)$.

PROOF. Applying Proposition 3.2 with $j=n$ and Proposition 3.3, we obtain $\mathcal{F}_{\alpha}(n) \subset$ $A_{\beta, n}^{1}\left(B_{n}\right) \subset \mathcal{F}_{\beta}(n)$ for $0 \leq \alpha<\beta$.

\section{Families $\mathcal{F}_{\alpha}(\boldsymbol{n})$ and radial derivatives}

The proof of the following proposition is similar to that of [6, Proposition 4.2].

Proposition 4.1. Suppose that $\alpha \geq 0, n \in \mathbb{N}$ and $f \in \mathcal{H}$ ol $\left(B_{n}\right)$. Then $f \in \mathcal{F}_{\alpha}(n)$ if and only if $\mathcal{R} f \in \mathcal{F}_{\alpha+1}(n)$.

Proof. Let $f \in \mathcal{F}_{\alpha}(n)$. Assume that $\alpha=0$. Then

$$
f(z)=f(0)+\int_{B_{n}} \log \frac{1}{1-\langle z, w\rangle} d \mu(w), \quad z \in B_{n},
$$

for some $\mu \in \mathcal{M}\left(B_{n}\right)$. Direct calculations show that

$$
\mathcal{R} f(z)=\int_{B_{n}} \frac{\langle z, w\rangle}{1-\langle z, w\rangle} d \mu(w)=F_{1}[\rho](z), \quad z \in B_{n},
$$

where $\rho=\mu-\mu\left(B_{n}\right) \nu_{n}$. So $\mathcal{R} f \in \mathcal{F}_{1}(n)$.

Now assume that $\alpha>0$. Then

$$
f(z)=\int_{B_{n}} \frac{1}{(1-\langle z, w\rangle)^{\alpha}} d \mu(w), \quad z \in B_{n},
$$

for some $\mu \in \mathcal{M}\left(B_{n}\right)$. We have

$$
\mathcal{R} f(z)=\int_{B_{n}} \frac{\alpha}{(1-\langle z, w\rangle)^{\alpha+1}} d \mu(w)-\alpha f(z), \quad z \in B_{n} .
$$

Note that $\alpha f \in \mathcal{F}_{\alpha}(n) \subset \mathcal{F}_{\alpha+1}(n)$ by Corollary 3.4, thus $\mathcal{R} f \in \mathcal{F}_{\alpha+1}(n)$. 
It is convenient to prove the converse implication in two steps.

Step 1. $\alpha=m \in \mathbb{N} \cup\{0\}$. By the hypothesis,

$$
\mathcal{R} f(z)=\int_{B_{n}} \frac{1}{(1-\langle z, w\rangle)^{m+1}} d \mu(w), \quad z \in B_{n} .
$$

Applying (2.1), we obtain

$$
f(z)-f(0)=\int_{0}^{1} \frac{\mathcal{R} f(t z)}{t} d t=\int_{0}^{1} \int_{B_{n}} \frac{1}{t(1-t\langle z, w\rangle)^{m+1}} d \mu(w) d t, \quad z \in B_{n} .
$$

If $\lambda \in \mathbb{C}$ and $|\lambda|<1$, then

$$
\frac{1}{t(1-t \lambda)^{m+1}}=\frac{1}{t}+\sum_{j=1}^{m+1} \frac{\lambda}{(1-t \lambda)^{j}}
$$

Put $\lambda=\langle z, w\rangle$. Note that $\mathcal{R} f(0)=0$, thus $\mu\left(B_{n}\right)=0$. Hence, changing the order of integration, we obtain

$$
\begin{aligned}
& f(z)-f(0)= \sum_{j=1}^{m+1} \int_{B_{n}} \int_{0}^{1} \frac{\langle z, w\rangle}{(1-t\langle z, w\rangle)^{j}} d t d \mu(w) \\
&= \int_{B_{n}} \log \frac{1}{1-\langle z, w\rangle} d \mu(w) \\
& \quad+\sum_{j=2}^{m+1} \int_{B_{n}} \frac{1}{(j-1)(1-\langle z, w\rangle)^{j-1}} d \mu(w) \\
& \in \mathcal{F}_{0}(n)+\cdots+\mathcal{F}_{m}(n) \subset \mathcal{F}_{m}(n)
\end{aligned}
$$

by Corollary 3.4. Recall that $1 \in \mathcal{F}_{m}(n)$, hence $f \in \mathcal{F}_{m}(n)$.

Step 2. $\alpha>0, \alpha \notin \mathbb{N}$. Repeating the arguments used in Step 1 and changing the order of integration,

$$
\begin{aligned}
f(z)-f(0)= & \int_{0}^{1} \int_{B_{n}} \frac{1}{t(1-t\langle z, w\rangle)^{\alpha+1}} d \mu(w) d t \\
= & \int_{B_{n}} \int_{0}^{1} \frac{\langle z, w\rangle}{(1-t\langle z, w\rangle)^{\alpha+1}} d t d \mu(w) \\
& \quad+\int_{0}^{1} \frac{1}{t} \int_{B_{n}} \frac{1}{(1-t\langle z, w\rangle)^{\alpha}} d \mu(w) d t .
\end{aligned}
$$

The inner integral in the first summand is explicitly calculable. So, consider the second summand. Put $[\alpha]=m \in \mathbb{N} \cup\{0\}$. We have $m+1>\alpha$, hence, by Corollary 3.4, there exists a measure $\rho \in \mathcal{M}\left(B_{n}\right)$ such that $F_{m+1}[\rho]=F_{\alpha}[\mu]$. Also, we have

$$
\rho\left(B_{n}\right)=F_{m+1}[\rho](0)=F_{\alpha}[\mu](0)=\mu\left(B_{n}\right)=0 .
$$


Therefore,

$$
\begin{aligned}
f(z)-f(0) & =\frac{1}{\alpha} \int_{B_{n}} \frac{1}{(1-\langle z, w\rangle)^{\alpha}} d \mu(w)+\int_{0}^{1} \int_{B_{n}} \frac{1}{t(1-t\langle z, w\rangle)^{m+1}} d \rho(w) d t \\
& \in \mathcal{F}_{\alpha}(n)+\mathcal{F}_{m}(n) .
\end{aligned}
$$

The latter property is obtained in Step 1. Recall that $\alpha>m$, hence $f \in \mathcal{F}_{\alpha}(n)+$ $\mathcal{F}_{m}(n) \subset \mathcal{F}_{\alpha}(n)$ by Corollary 3.4.

TheOREM 4.2. Assume that $\alpha \geq 0, n \in \mathbb{N}$ and $f \in \mathcal{H} \operatorname{lol}\left(B_{n}\right)$. Then the following properties are equivalent:

(i) $f \in \mathcal{F}_{\alpha}(n)$;

(ii) $\mathcal{R} f \in \mathcal{F}_{\alpha+1}(n)$;

(iii) $R f \in \mathcal{F}_{\alpha+1}(n)$.

Proof. Proposition 4.1 guarantees that (i) holds if and only if (ii) holds. Next, (i) and (ii) imply (iii). Finally, assume that (iii) holds. Then

$$
R f \in \mathcal{F}_{\alpha+1}(n) \subset \mathcal{F}_{\alpha+3 / 2}(n) \subset A_{\alpha+1, n-1}^{1}\left(B_{n}\right)
$$

by Proposition 3.2(ii) with $j=n-1$. Therefore, $f \in A_{\alpha+1, n}^{1}\left(B_{n}\right) \subset \mathcal{F}_{\alpha+1}(n)$ by Proposition 3.2(i). So $\mathcal{R} f=R f-f \in \mathcal{F}_{\alpha+1}(n)$, that is, (iii) implies (ii).

\section{Spaces $\mathcal{F}_{\alpha}(n)$ and $\mathcal{K}_{\alpha}(n)$}

It is shown in [6] that analogs of Proposition 3.2, Corollary 3.4 and Theorem 4.2 hold for the spaces $\mathcal{K}_{\alpha}(n)$ of fractional Cauchy transforms. Note that the arguments used in Sections 3 and 4 provide alternative proofs of those analogs. Also, the following assertion implies certain embedding properties.

Proposition 5.1. Let $n \in \mathbb{N}$ and let $\alpha \geq 0$. Then $\mathcal{F}_{\alpha}(n) \subset \mathcal{K}_{\alpha}(n)$.

ProOF. Suppose that $\alpha>0, \mu \in \mathcal{M}\left(B_{n}\right)$ and

$$
f(z)=\int_{B_{n}} \frac{d \mu(w)}{(1-\langle z, w\rangle)^{\alpha}}, \quad z \in B_{n} .
$$

Note that $\mu \in \mathcal{M}\left(\bar{B}_{n}\right)$, where $\mathcal{M}\left(\bar{B}_{n}\right)$ denotes the space of complex-valued Borel measures defined on the closed ball $\bar{B}_{n}$. Without loss of generality, assume that $\mu$ is a probability measure. Let $\delta_{\xi}$ denote the point mass at $\xi \in \mathbb{C}^{n}$. By the Banach-Alaoglu theorem, there exist probability measures $\mu_{k}=\sum_{j=1}^{J(k)} a_{k, j} \delta_{\xi_{k, j}}, \xi_{k, j} \in \bar{B}_{n}$, such that $\mu_{k} \rightarrow \mu$ in the weak* topology of $\mathcal{M}\left(\bar{B}_{n}\right)$. Therefore,

$$
\sum_{j=1}^{J(k)} \frac{a_{k, j}}{\left(1-\left\langle z, \xi_{j, k}\right\rangle\right)^{\alpha}} \rightarrow f(z), \quad \text { as } k \rightarrow \infty,
$$

for all $z \in B_{n}$. 
Let $k \in \mathbb{N}$. If $\xi_{k, j} \in \partial B_{n}$, then put $\rho_{k, j}=\delta_{\xi_{k, j}} \in \mathcal{M}\left(\partial B_{n}\right)$. Thus,

$$
\frac{1}{\left(1-\left\langle z, \xi_{k, j}\right\rangle\right)^{\alpha}}=K_{\alpha}\left[\rho_{k, j}\right](z), \quad z \in B_{n}
$$

Further, assume that $\xi_{k, j} \in B_{n}$. Given $z \in B_{n}$,

$$
h(w)=\frac{1}{(1-\langle z, w\rangle)^{\alpha}} \in \overline{\mathcal{H} o l}\left(B_{n}\right) \cap C\left(\bar{B}_{n}\right), \quad w \in B_{n} .
$$

Hence,

$$
\frac{1}{(1-\langle z, w\rangle)^{\alpha}}=\int_{\partial B_{n}} \frac{1}{(1-\langle z, \zeta\rangle)^{\alpha}} \frac{1-|w|^{2}}{|w-\zeta|^{2 n}} d \sigma_{n}(\zeta), \quad z, w \in B_{n}
$$

In other words, if $\xi_{k, j} \in B_{n}$, then

$$
\frac{1}{\left(1-\left\langle z, \xi_{k, j}\right\rangle\right)^{\alpha}}=K_{\alpha}\left[\rho_{k, j}\right](z), \quad z \in B_{n}
$$

for a probability measure $\rho_{k, j} \in \mathcal{M}\left(\partial B_{n}\right)$. Put

$$
\rho_{k}=\sum_{j=1}^{J(k)} a_{k, j} \rho_{k, j}
$$

Then $\rho_{k} \in \mathcal{M}\left(\partial B_{n}\right)$ is a probability measure and

$$
K_{\alpha}\left[\rho_{k}\right](z)=\sum_{j=1}^{J(k)} \frac{a_{k, j}}{\left(1-\left\langle z, \xi_{j, k}\right\rangle\right)^{\alpha}} \rightarrow f(z), \quad \text { as } k \rightarrow \infty,
$$

for all $z \in B_{n}$. By the Banach-Alaoglu theorem, there exists a subsequence $\rho_{k_{m}}$ such that $\rho_{k_{m}} \rightarrow \rho$ in the weak* topology of $\mathcal{M}\left(\partial B_{n}\right)$. Therefore,

$$
K_{\alpha}\left[\rho_{k_{m}}\right](z) \rightarrow K_{\alpha}[\rho](z), \quad \text { as } k_{m} \rightarrow \infty,
$$

for all $z \in B_{n}$. Hence, $f=K_{\alpha}[\rho]$ by (5.1).

The proof for $\alpha=0$ is analogous.

Below we show that $\mathcal{F}_{\alpha}(n) \neq \mathcal{K}_{\alpha}(n)$ for all $\alpha \geq 0$.

Given $\xi \in \partial B_{n}$ and $C>1$, recall that the Korányi approach region $D_{C}(\xi)$ is defined by the identity

$$
D_{C}(\xi)=\left\{z \in B_{n}:|1-\langle z, \xi\rangle|<C(1-|z|)\right\} .
$$

Proposition 5.2. Suppose that $\alpha>0, n \in \mathbb{N}, \mu \in \mathcal{M}\left(B_{n}\right), \xi \in \partial B_{n}$ and $C>1$. Then

$$
\lim _{\substack{z \rightarrow \xi \\ z \in D_{C}(\xi)}}(1-\langle z, \xi\rangle)^{\alpha} F_{\alpha}[\mu](z)=0 .
$$


Proof. Assume that $w \in B_{n}$ and $\xi \in \partial B_{n}$. Then

$$
\lim _{\substack{z \rightarrow \xi \\ z \in B_{n}}} \frac{(1-\langle z, \xi\rangle)^{\alpha}}{(1-\langle z, w\rangle)^{\alpha}}=0
$$

If $z \in D_{C}(\xi)$, then

$$
\left|\frac{1-\langle z, \xi\rangle}{1-\langle z, w\rangle}\right|^{\alpha} \leq \frac{|1-\langle z, \xi\rangle|^{\alpha}}{(1-|z|)^{\alpha}} \leq C^{\alpha}
$$

Hence,

$$
\lim _{\substack{z \rightarrow \xi \\ z \in D_{C}(\xi)}}(1-\langle z, \xi\rangle)^{\alpha} F_{\alpha}[\mu](z)=\lim _{\substack{z \rightarrow \xi \\ z \in D_{C}(\xi)}} \int_{B_{n}} \frac{(1-\langle z, \xi\rangle)^{\alpha}}{(1-\langle z, w\rangle)^{\alpha}} d \mu(w)=0
$$

by the dominated convergence theorem.

Assume that $\alpha>0, n \in \mathbb{N}, \rho \in \mathcal{M}\left(\partial B_{n}\right), \xi \in \partial B_{n}$ and $C>1$. Then, by [6, Proposition 6.1],

$$
\lim _{\substack{z \rightarrow \xi \\ z \in D_{C}(\xi)}}(1-\langle z, \xi\rangle)^{\alpha} K_{\alpha}[\rho](z)=\rho(\{\xi\}) .
$$

Therefore, if $\rho(\{\xi\}) \neq 0$ for some point $\xi \in \partial B_{n}$, then $K_{\alpha}[\rho] \in \mathcal{K}_{\alpha}(n) \backslash \mathcal{F}_{\alpha}(n)$. Similar arguments show that $\mathcal{F}_{0}(n) \neq \mathcal{K}_{0}(n)$.

\section{Multipliers: necessary conditions}

Proposition 6.1. Assume that $n \in \mathbb{N}, 0 \leq \alpha \leq \beta$ and $g \in \mathcal{H} \operatorname{lol}\left(B_{n}\right)$. Then the following properties are equivalent.

(i) $\quad g \in \mathfrak{M}\left(\mathcal{K}_{\alpha}(n), \mathcal{K}_{\beta}(n)\right)$.

(ii) $g \in \mathfrak{M}\left(\mathcal{F}_{\alpha}(n), \mathcal{K}_{\beta}(n)\right)$.

(iii) $g(z) k_{\alpha}(\langle z, w\rangle) \in \mathcal{K}_{\beta}(n)$ for all $w \in B_{n}$, and

$$
\sup _{w \in B_{n}}\left\|g(z) k_{\alpha}(\langle z, w\rangle)\right\|_{\mathcal{K}_{\beta}(n)} \leq C<\infty
$$

if $\alpha=0$, then it is also required that $g \in \mathcal{K}_{\beta}(n)$.

(iv) $g(z) k_{\alpha}(\langle z, \zeta\rangle) \in \mathcal{K}_{\beta}(n)$ for all $\zeta \in \partial B_{n}$, and

$$
\sup _{\zeta \in \partial B_{n}}\left\|g(z) k_{\alpha}(\langle z, \zeta\rangle)\right\|_{\mathcal{K}_{\beta}(n) \leq C<\infty}
$$

if $\alpha=0$, then it is also required that $g \in \mathcal{K}_{\beta}(n)$.

Proof. By Proposition 5.1, (i) implies (ii). Let (ii) hold. Note that

$$
\left\|k_{\alpha}(\langle z, w\rangle)\right\|_{\mathcal{F}_{\alpha}(n)} \leq 1, \quad \forall w \in B_{n},
$$


hence (iii) holds by the closed graph theorem. If $\alpha=0$, then $g \in \mathcal{K}_{\beta}(n)$, since $1 \in \mathcal{F}_{0}(n)$.

Now, let (iii) hold and let $\zeta \in \partial B_{n}$. Fix a sequence $\left\{w_{j}\right\}_{j=1}^{\infty} \subset B_{n}$ such that $w_{j} \rightarrow \zeta$. Note that

$$
g(z) k_{\alpha}\left(\left\langle z, w_{j}\right\rangle\right) \rightarrow g(z) k_{\alpha}(\langle z, \zeta\rangle), \quad \text { as } j \rightarrow \infty,
$$

for all $z \in B_{n}$. By property (iii), there exist measures $\rho_{j} \in \mathcal{M}\left(\partial B_{n}\right)$ such that $\left\|\rho_{j}\right\|_{\mathcal{M}\left(\partial B_{n}\right)} \leq C$ and

$$
g(z) k_{\alpha}\left(\left\langle z, w_{j}\right\rangle\right)=\int_{\partial B_{n}} k_{\beta}(\langle z, \zeta\rangle) d \rho_{j}(\zeta), \quad z \in B_{n} .
$$

By the Banach-Alaoglu theorem, there exists a subsequence $\rho_{j_{m}}$ such that $\rho_{j_{m}} \rightarrow \rho$ in the weak* topology of $\mathcal{M}\left(\partial B_{n}\right)$. Note that $\|\rho\|_{\mathcal{M}\left(\partial B_{n}\right)} \leq C$. So

$$
g(z) k_{\alpha}\left(\left\langle z, w_{j_{m}}\right\rangle\right) \rightarrow \int_{\partial B_{n}} k_{\beta}(\langle z, \zeta\rangle) d \rho(\zeta), \quad \text { as } j_{m} \rightarrow \infty,
$$

for all $z \in B_{n}$. By (6.1), we obtain

$$
\left\|g(z) k_{\alpha}(\langle z, \zeta\rangle)\right\|_{\mathcal{K}_{\beta}(n)} \leq\|\rho\|_{\mathcal{M}\left(\partial B_{n}\right)} \leq C .
$$

Finally, standard arguments, based on approximation by discrete measures, show that (iv) implies (i) (see [11, Theorem 1], [7, Lemma 2.1]).

Corollary 6.2. Assume that $n \in \mathbb{N}$ and $0 \leq \alpha \leq \beta$. Then

$$
\mathfrak{M}\left(\mathcal{F}_{\alpha}(n), \mathcal{F}_{\beta}(n)\right) \subset \mathfrak{M}\left(\mathcal{K}_{\alpha}(n), \mathcal{K}_{\beta}(n)\right) .
$$

In particular, $\mathfrak{M}_{\alpha}(n) \subset \mathfrak{M}\left(\mathcal{K}_{\alpha}(n)\right)$.

Proof. We have $\mathcal{F}_{\beta}(n) \subset \mathcal{K}_{\beta}(n)$, hence

$$
\mathfrak{M}\left(\mathcal{F}_{\alpha}(n), \mathcal{F}_{\beta}(n)\right) \subset \mathfrak{M}\left(\mathcal{F}_{\alpha}(n), \mathcal{K}_{\beta}(n)\right)=\mathfrak{M}\left(\mathcal{K}_{\alpha}(n), \mathcal{K}_{\beta}(n)\right)
$$

by Proposition 6.1 .

Standard arguments guarantee that $\mathfrak{M}_{\alpha}(n) \subset H^{\infty}\left(B_{n}\right)$ for all $\alpha \geq 0$. Moreover, if $g \in \mathfrak{M}_{\alpha}(n), \alpha>0, n \in \mathbb{N}$, then

$$
\sup _{\xi \in \partial B_{n}} \int_{0}^{1}|\mathcal{R} g(r \xi)| d r<+\infty
$$

by Proposition 6.1 and [6, Proposition 7.3].

Corollary 6.3. Suppose that $n \in \mathbb{N}, M \in\{1, \ldots, n\}, n \geq \alpha>n-M$ and that $g \in \mathfrak{M}_{\alpha}(n)$. Then

$$
\sup _{\zeta \in \partial B_{n}} \int_{B_{n}} \frac{\left|R^{k} g(z)\right|(1-|z|)^{\alpha+M-n-1}}{|1-\langle z, \zeta\rangle|^{\alpha+M-k}}\left(\log \frac{e}{1-|z|}\right)^{-1-\varepsilon} d v_{n}(z)<\infty
$$

for $k=1, \ldots, M$ and any $\varepsilon>0$.

PROOF. We apply Proposition 6.1 and [5, Theorem 1.2]. 


\section{Multipliers: sufficient conditions}

Suppose that $n \in \mathbb{N}, \alpha \geq 0$ and $g \in \mathfrak{M}_{\alpha}(n)$. Note that $g \in H^{\infty}\left(B_{n}\right)$, hence

$$
\sup _{w \in B_{n}}\left\|(g(z)-g(w)) k_{\alpha}(\langle z, w\rangle)\right\|_{\mathcal{F}_{\alpha}(n)}<\infty
$$

by the closed graph theorem.

7.1. Spaces $\mathfrak{M}_{\alpha}(\boldsymbol{n})$ with $\boldsymbol{\alpha}>\boldsymbol{n}$. The following proposition shows that the necessary condition (7.1) becomes a sufficient one, for $\alpha>n$, when the norm of $\mathcal{F}_{\alpha}(n)$ is replaced by the norm of the weighted Bergman space $A_{\alpha-n}^{1}\left(B_{n}\right)$.

Proposition 7.1. Assume that $n \in \mathbb{N}, \alpha>n$ and $g \in H^{\infty}\left(B_{n}\right)$. Suppose that

$$
\sup _{w \in B_{n}}\left\|\frac{g(z)-g(w)}{(1-\langle z, w\rangle)^{\alpha}}\right\|_{A_{\alpha-n}^{1}\left(B_{n}\right)} \leq C<\infty .
$$

Then $g \in \mathfrak{M}_{\alpha}(n)$.

We will need two auxiliary lemmas.

Lemma 7.2. Assume that $\mu$ and $\mu_{k}, k \in \mathbb{N}$, are probability measures on $B_{n}$. Suppose that $\mu_{k} \rightarrow \mu$ in the weak ${ }^{*}$ topology of $\mathcal{M}\left(B_{n}\right)$. Let $\varepsilon>0$. Then there exists an $r=r(\varepsilon) \in(0,1)$ such that $\mu\left(B_{n} \backslash r B_{n}\right)<\varepsilon$ and $\mu_{k}\left(B_{n} \backslash r B_{n}\right)<\varepsilon$ for all $k \in \mathbb{N}$.

PROOF. We have $\mu\left(B_{n}\right)<\infty$, hence $\mu\left(B_{n} \backslash R B_{n}\right)<\varepsilon$ for some $R \in(0,1)$. Put $r=$ $(1+R) / 2$ and consider a function $f \in C_{0}\left(r B_{n}\right)$ such that $0 \leq f \leq 1$ and $\left.f\right|_{R B_{n}} \equiv 1$. By the definition of weak* convergence, we obtain

$$
\mu_{k}\left(r B_{n}\right) \geq \int_{B_{n}} f d \mu_{k} \rightarrow \int_{B_{n}} f d \mu \geq \mu\left(R B_{n}\right)>1-\varepsilon .
$$

Therefore, $\mu_{k}\left(B_{n} \backslash r B_{n}\right)<\varepsilon$ for all sufficiently large $k$. Hence, the required property holds.

Lemma 7.3. Let $\mu_{k} \in \mathcal{M}\left(B_{n}\right), k \in \mathbb{N}$. Assume that, for any $\varepsilon>0$, there exists an $r \in(0,1)$ such that $\left|\mu_{k}\right|\left(B_{n} \backslash r B_{n}\right)<\varepsilon$ for all $k \in \mathbb{N}$. Let $\mu_{k} \rightarrow \mu$ in the weak* topology of $\mathcal{M}\left(B_{n}\right)$. Then

$$
\int_{B_{n}} f d \mu_{k} \rightarrow \int_{B_{n}} f d \mu, \quad \text { as } k \rightarrow \infty,
$$

for any bounded function $f \in C\left(B_{n}\right)$.

Proof. Assume that $f \in C\left(B_{n}\right)$ and $|f(z)| \leq 1$ for all $z \in B_{n}$.

Fix an $\varepsilon>0$. We have $|\mu|\left(B_{n}\right)<\infty$, thus, applying the hypotheses of the lemma, choose $r \in(0,1)$ such that $|\mu|\left(B_{n} \backslash r B_{n}\right)<\varepsilon$ and $\left|\mu_{k}\right|\left(B_{n} \backslash r B_{n}\right)<\varepsilon$ for 
all $k \in \mathbb{N}$. Consider a function $f_{0} \in C_{0}\left(B_{n}\right)$ such that $f_{0}(z)=f(z)$ for all $z \in r B_{n}$, and $|f(z)| \leq 1$ for all $z \in B_{n}$. By the definition of the weak* topology of $\mathcal{M}\left(B_{n}\right)$,

$$
\left|\int_{B_{n}} f_{0} d \mu_{k}-\int_{B_{n}} f_{0} d \mu\right|<\varepsilon
$$

for all $k \geq k_{0}$. Also note that

$$
\int_{B_{n}}\left|f-f_{0}\right| d\left(\left|\mu_{k}\right|+|\mu|\right) \leq 2 \int_{B_{n} \backslash r B_{n}} d\left(\left|\mu_{k}\right|+|\mu|\right)<4 \varepsilon .
$$

Therefore,

$$
\left|\int_{B_{n}} f d \mu_{k}-\int_{B_{n}} f d \mu\right|<5 \varepsilon
$$

for all $k \geq k_{0}$.

Proof of Proposition 7.1. Let $f=F_{\alpha}[\mu], \alpha>n$. We have to prove that $f g \in$ $\mathcal{F}_{\alpha}(n)$. Without loss of generality, assume that $\mu \in \mathcal{M}\left(B_{n}\right)$ is a probability measure. Applying the Banach-Alaoglu theorem, select a sequence of probability measures $\mu_{k}=\sum_{j=1}^{J(k)} a_{k, j} \delta_{w_{k, j}}, w_{k, j} \in B_{n}$, such that $\mu_{k} \rightarrow \mu$ in the weak* topology of $\mathcal{M}\left(B_{n}\right)$.

If $z \in B_{n}$, then $(1-\langle z, \cdot\rangle)^{-\alpha} \in C\left(\bar{B}_{n}\right)$. Hence, Lemmas 7.2 and 7.3 guarantee that

$$
g(z) \sum_{j=1}^{J(k)} \frac{a_{k, j}}{\left(1-\left\langle z, w_{k, j}\right\rangle\right)^{\alpha}} \rightarrow g(z) \int_{B_{n}} \frac{d \mu(w)}{(1-\langle z, w\rangle)^{\alpha}}=g(z) f(z)
$$

for all $z \in B_{n}$. Consider the measures $\rho_{k}=\sum_{j=1}^{J(k)} a_{k, j} g\left(w_{k, j}\right) \delta_{w_{k, j}}$. Note that $g \in$ $H^{\infty}\left(B_{n}\right)$ and the measures $\mu_{k} \in \mathcal{M}\left(B_{n}\right)$ satisfy the hypotheses of Lemma 7.3, thus the measures $\rho_{k} \in \mathcal{M}\left(B_{n}\right)$ also satisfy the hypotheses of Lemma 7.3.

Remark that $\left\|\rho_{k}\right\| \leq\|g\|_{H^{\infty}\left(B_{n}\right)}$, thus, by the Banach-Alaoglu theorem, there exists a subsequence $\rho_{k_{m}}$ which converges in the weak* topology. Without loss of generality, assume that $\rho_{k} \rightarrow \rho$ in the weak* topology of $\mathcal{M}\left(B_{n}\right)$. Lemma 7.3 guarantees that

$$
\sum_{j=1}^{J(k)} \frac{a_{k, j} g\left(w_{k, j}\right)}{\left(1-\left\langle z, w_{k, j}\right\rangle\right)^{\alpha}} \rightarrow \int_{B_{n}} \frac{d \rho(w)}{(1-\langle z, w\rangle)^{\alpha}}, \quad \text { as } k \rightarrow \infty,
$$

for all $z \in B_{n}$. Put

$$
h_{k}(z)=\sum_{j=1}^{J(k)} \frac{a_{k, j}\left(g(z)-g\left(w_{k, j}\right)\right)}{\left(1-\left\langle z, w_{k, j}\right\rangle\right)^{\alpha}} .
$$

Recall that $a_{k, j}>0$ and $\sum_{j=1}^{J(k)} a_{k, j}=1$, hence $\left\|h_{k}\right\|_{A_{\alpha-n}^{1}\left(B_{n}\right)} \leq C$ by (7.2). Therefore, the sequence $\left\{h_{k}\right\}_{k=1}^{\infty}$ is uniformly bounded on compact subsets of the ball $B_{n}$. Thus, there exists a subsequence which converges to a holomorphic function uniformly on 
compact subsets. Without loss of generality, assume that $h_{k}(z) \rightarrow h(z) \in \mathcal{H} \operatorname{Hol}\left(B_{n}\right)$ for all $z \in B_{n}$. Fatou's theorem guarantees that $h \in A_{\alpha-n}^{1}\left(B_{n}\right)$.

Consider the limit as $k \rightarrow \infty$ in identity (7.5). Applying (7.3) and (7.4), we obtain

$$
f(z) g(z)=\int_{B_{n}} \frac{d \rho(w)}{(1-\langle z, w\rangle)^{\alpha}}+h(z), \quad z \in B_{n} .
$$

Recall that $A_{\alpha-n}^{1}\left(B_{n}\right) \subset \mathcal{F}_{\alpha}(n)$ by Proposition 3.1(i), thus $f g \in \mathcal{F}_{\alpha}(n)$.

Let $\delta>0$. The holomorphic Lipschitz space $\Lambda_{\delta}\left(B_{n}\right)$ consists of those $g \in \mathcal{H} \operatorname{col}\left(B_{n}\right)$ for which

$$
\left|R^{j} g(z)\right| \leq C(1-|z|)^{\delta-j}, \quad z \in B_{n},
$$

where $j$ is the least integer such that $j>\delta$. It is well known that, replacing $R$ by $\mathcal{R}$ in estimate (7.6), we obtain an equivalent definition of the space $\Lambda_{\delta}\left(B_{n}\right)$. In particular, if $0<\delta<1$, then the space $\Lambda_{\delta}\left(B_{n}\right)$ is defined by the following property:

$$
|\mathcal{R} g(z)| \leq C(1-|z|)^{\delta-1}, \quad z \in B_{n} .
$$

We will need the following lemma.

Lemma 7.4 [4, Lemma 2.7]. Assume that $n \in \mathbb{N}, 0<\delta<1$ and $g \in \Lambda_{\delta}\left(B_{n}\right)$. Then $g$ extends continuously to the closed ball $\bar{B}_{n}$. Moreover, if $0<\tau<\min \{1 / 2, \delta\}$, then there exists a constant $C>0$ such that

$$
|g(z)-g(w)| \leq C|1-\langle z, w\rangle|^{\tau} \quad \forall z, w \in \bar{B}_{n} .
$$

Corollary 7.5. Suppose that $n \in \mathbb{N}, \alpha>n$ and $\delta>0$. Then $\Lambda_{\delta}\left(B_{n}\right) \subset \mathfrak{M}_{\alpha}(n)$.

PROOF. Without loss of generality, assume that $0<\delta<1 / 2$. Let $g \in \Lambda_{\delta}\left(B_{n}\right)$. Lemma 7.4 guarantees that inequality (7.7) holds for some $\tau \in(0,1 / 2)$. Hence,

$$
\begin{aligned}
& \sup _{w \in B_{n}} \int_{B_{n}} \frac{|g(z)-g(w)|}{|1-\langle z, w\rangle|^{\alpha}}(1-|z|)^{\alpha-n-1} d v_{n}(z) \\
& \quad \leq \sup _{w \in B_{n}} \int_{B_{n}} \frac{(1-|z|)^{\alpha-n-1}}{|1-\langle z, w\rangle|^{\alpha-\tau}} d v_{n}(z) \\
& \quad \leq C
\end{aligned}
$$

by [10, Proposition 1.4.10]. It remains to apply Proposition 7.1.

\subsection{Spaces $\mathfrak{M}_{\alpha}(n)$ with $0 \leq \alpha \leq n$.}

Proposition 7.6. Assume that $n \in \mathbb{N}, \alpha \geq 0, \quad \beta>\max \{\alpha, n\}$ and $g \in \mathcal{H} \operatorname{Hol}\left(B_{n}\right)$. Suppose that

$$
\sup _{w \in B_{n}}\left\|g(z) k_{\alpha}(\langle z, w\rangle)\right\|_{A_{\beta-n}^{1}\left(B_{n}\right)}<\infty .
$$

If $\alpha=0$, then suppose also that $g \in A_{\beta-n}^{1}\left(B_{n}\right)$. Then $g \in \mathfrak{M}\left(\mathcal{F}_{\alpha}(n), A_{\beta-n}^{1}\left(B_{n}\right)\right)$. 
PROOF. If $\alpha>0$, then it suffices to repeat the arguments used in the proof of Proposition 7.1, putting $\rho_{k}=\rho=0$. Now, let $\alpha=0$ and let $f=f(0)+F_{0}[\mu]$, $\mu \in \mathcal{M}\left(B_{n}\right)$. As in the case $\alpha>0$, we have $F_{0}[\mu] \cdot g \in A_{\beta-n}^{1}\left(B_{n}\right)$. It remains to remark that $f(0) g \in A_{\beta-n}^{1}\left(B_{n}\right)$.

Theorem 7.7. Let $n \in \mathbb{N}$ and let $g \in \Lambda_{\delta}\left(B_{n}\right)$ for some $\delta>0$. Assume that $M \in$ $\{1, \ldots, n\}$ and $n \geq \alpha>n-M$, or assume that $M=n+1$ and $\alpha=0$. Suppose that

$$
\sup _{w \in B_{n}} \int_{B_{n}} \frac{\left|R^{k} g(z)\right|(1-|z|)^{\alpha+M-n-1}}{|1-\langle z, w\rangle|^{\alpha+M-k}} d v_{n}(z)<\infty, \quad k=1, \ldots, \min \{n, M\} .
$$

If $\alpha=0$ and $M=n+1$, then suppose also that

$$
\int_{B_{n}}\left|R^{n+1} g(z)\right| \log \frac{e}{1-|z|} d \nu_{n}(z)<\infty .
$$

Then $g \in \mathfrak{M}_{\alpha}(n)$.

ProOF. Let $f \in \mathcal{F}_{\alpha}(n)$. Theorem 4.2 guarantees that $\mathcal{R}^{M} f \in \mathcal{F}_{\alpha+M}(n)$. We have $g \in \Lambda_{\delta}\left(B_{n}\right) \subset \mathfrak{M}_{\alpha+M}(n)$ by Corollary 7.5 , hence

$$
\mathcal{R}^{M} f \cdot g \in \mathcal{F}_{\alpha+M}(n) .
$$

Further, let $k \in\{1, \ldots, \min \{n, M\}\}$. Then $\mathcal{R}^{M-k} f \in \mathcal{F}_{\alpha+M-k}(n)$ by Theorem 4.2. Note that $\alpha+M>\max \{n, \alpha+M-k\}$, hence property (7.9) and Proposition 7.6 guarantee that

$$
\mathcal{R}^{M-k} f \cdot R^{k} g \in A_{\alpha+M-n}^{1}\left(B_{n}\right) \subset \mathcal{F}_{\alpha+M}(n)
$$

by Proposition 3.1(i).

If $\alpha=0$ and $M=n+1$, then (7.10) implies (7.8) for $R^{n+1} g, \alpha=0$ and $\beta=$ $n+1$. Also, property (7.10) guarantees that $R^{n+1} g \in A_{1}^{1}\left(B_{n}\right)$. Hence, applying Proposition 7.6 with $\beta=n+1$, we obtain property (7.12) for $\alpha=0$ and $k=M=$ $n+1$.

Now, properties (2.2), (7.11) and (7.12) guarantee that $R^{M}(f g) \in \mathcal{F}_{\alpha+M}(n)$. Finally, $f g \in \mathcal{F}_{\alpha}(n)$ by Theorem 4.2.

Assume that $g \in \mathcal{H o l}\left(B_{n}\right), \quad M \in\{1, \ldots, n\}$ and $n \geq \alpha>n-M$. Note that condition (7.9) is equivalent to the following property:

$$
\sup _{\zeta \in \partial B_{n}} \int_{B_{n}} \frac{\left|R^{k} g(z)\right|(1-|z|)^{\alpha+M-n-1}}{|1-\langle z, \zeta\rangle|^{\alpha+M-k}} d v_{n}(z)<\infty, \quad k=1, \ldots, M .
$$

Indeed, (7.9) implies (7.13) by Fatou's theorem. On the other hand, if $\lambda \in B_{1}$ and $r \in[0,1)$, then $|1-\lambda| \leq 4|1-r \lambda|$. Hence, $|1-\langle z, \zeta\rangle| \leq 4|1-\langle z, r \zeta\rangle|$ for all $z \in B_{n}, \zeta \in \partial B_{n}$ and $r \in[0,1)$. Therefore, (7.13) implies (7.9). 
Note that the sufficient condition in Theorem 7.7, for $\alpha>0$, is not too far from the necessary condition in Corollary 6.3. Indeed, dropping the logarithmic term in (6.2), we obtain (7.13) or, equivalently, (7.9).

Several explicit conditions, sufficient for (7.13), are given in [4,5] when $n>\alpha>0$. As an illustration, consider the holomorphic Lipschitz spaces $\Lambda_{\delta}\left(B_{n}\right), \delta>0$.

Corollary 7.8. Let $n \in \mathbb{N}$. Assume that $0 \leq \alpha \leq n$ and $g \in \Lambda_{\delta}\left(B_{n}\right)$ for some $\delta>n-\alpha$. Then $g \in \mathfrak{M}_{\alpha}(n)$.

PROOF. If $0<\alpha<n$ and $g \in \Lambda_{\delta}\left(B_{n}\right)$ for some $\delta>n-\alpha$, then, as shown in the proof of [5, Corollary 4.2], property (7.13) holds. Hence, Theorem 7.7 is applicable.

Further, let $\alpha=0$ and let $g \in \Lambda_{\delta}\left(B_{n}\right)$ for some $\delta \in(n, n+1)$. Put $M=n+1$. Then

$$
\left|R^{n+1} g(z)\right| \leq C(1-|z|)^{\delta-n-1}, \quad z \in B_{n} .
$$

Therefore, property (7.10) holds. For $k=1, \ldots, n$, we have $R^{k} g \in H^{\infty}\left(B_{n}\right)$, thus

$$
\sup _{w \in B_{n}} \int_{B_{n}} \frac{\left|R^{k} g(z)\right|(1-|z|)^{\alpha+M-n-1}}{|1-\langle z, w\rangle|^{\alpha+M-k}} d v_{n}(z) \leq \sup _{w \in B_{n}} \int_{B_{n}} \frac{d v_{n}(z)}{|1-\langle z, w\rangle|^{n+1-k}} \leq C
$$

by [10, Proposition 1.4.10]. So, $g \in \mathfrak{M}_{0}(n)$ by Theorem 7.7.

Finally, let $\alpha=n$ and let $g \in \Lambda_{\delta}\left(B_{n}\right)$ for some $\delta \in(0,1)$. Put $M=1$. Then

$$
\sup _{w \in B_{n}} \int_{B_{n}} \frac{|R g(z)|(1-|z|)^{\alpha+M-n-1}}{|1-\langle z, w\rangle|^{\alpha+M-1}} d v_{n}(z) \leq C \int_{B_{n}} \frac{(1-|z|)^{\delta-1}}{|1-\langle z, w\rangle|^{n}} d v_{n}(z) \leq C
$$

by [10, Proposition 1.4.10]. So, $g \in \mathfrak{M}_{n}(n)$ by Theorem 7.7 .

\section{References}

[1] M. Andersson, M. Passare and R. Sigurdsson, Complex Convexity and Analytic Functionals, Progress in Mathematics, 225 (Birkhäuser, Basel, 2004).

[2] F. Beatrous and J. Burbea, 'Holomorphic Sobolev spaces on the ball', Dissertationes Math. (Rozprawy Mat.) 276 (1989), 60.

[3] J. A. Cima, A. L. Matheson and W. T. Ross, The Cauchy Transform, Mathematical Surveys and Monographs, 125 (American Mathematical Society, Providence, RI, 2006).

[4] E. Doubtsov, 'Multipliers of fractional Cauchy transforms', Integral Equations Operator Theory 64(2) (2009), 177-192.

[5] E. Doubtsov, 'Fractional Cauchy transforms, multipliers and Cesàro operators', Proc. Amer. Math. Soc. 138(2) (2010), 663-673.

[6] E. S. Dubtsov, 'Families of fractional Cauchy transforms in the ball', Algebra i Analiz 21(6) (2009), 151-181 (in Russian); St. Petersburg Math. J., to appear (English translation).

[7] R. A. Hibschweiler and T. H. MacGregor, 'Multipliers of families of Cauchy-Stieltjes transforms', Trans. Amer. Math. Soc. 331(1) (1992), 377-394.

[8] R. A. Hibschweiler and T. H. MacGregor, Fractional Cauchy Transforms, Chapman \& Hall/CRC Monographs and Surveys in Pure and Applied Mathematics, 136 (Chapman \& Hall/CRC, Boca Raton, FL, 2006).

[9] H. T. Kaptanoğlu, 'Carleson measures for Besov spaces on the ball with applications', J. Funct. Anal. 250(2) (2007), 483-520. 
[10] W. Rudin, Function Theory in the Unit Ball of $\mathbf{C}^{n}$, Grundlehren Math. Wiss., 241 (Springer, New York, Berlin, 1980).

[11] S. A. Vinogradov, M. G. Goluzina and V. P. Havin, 'Multipliers and divisors of Cauchy-Stieltjes type integrals', Zap. Naučn. Sem. Leningrad. Otdel. Mat. Inst. Steklov. (LOMI) 19 (1970), 55-78 (in Russian); Seminars in Math., V. A. Steklov Math. Inst., Leningrad 19 (1972), 29-42 (English translation).

[12] K. Zhu, Spaces of Holomorphic Functions in the Unit Ball, Graduate Texts in Mathematics, 226 (Springer, New York, 2005).

EVGUENI DOUBTSOV, St. Petersburg Department of V.A. Steklov Mathematical Institute, Fontanka 27, St. Petersburg 191023, Russia

e-mail: dubtsov@pdmi.ras.ru 